\title{
Association mapping and genomic prediction for resistance to sudden death syndrome in early maturing soybean germplasm
}

\author{
Yong Bao · James E. Kurle • Grace Anderson • \\ Nevin D. Young
}

Received: 30 December 2014/ Accepted: 8 May 2015/Published online: 17 May 2015

(C) The Author(s) 2015. This article is published with open access at Springerlink.com

\begin{abstract}
Sudden death syndrome (SDS), caused by Fusarium virguliforme, has spread to northern soybean growing regions in the US causing significant yield losses. The objectives of this study were to identify loci underlying variation in plant responses to SDS through association mapping (AM) and to assess prediction accuracy of genomic selection (GS) in a panel of early maturing soybean germplasm. A set of 282 soybean breeding lines was selected from the University of Minnesota soybean breeding program and then genotyped using a genome-wide panel of 1536 single-nucleotide polymorphism markers. Four resistance traits, root lesion severity (RLS), foliar symptom severity (FSS), root retention (RR), and dry matter reduction (DMR), were evaluated using soil inoculation in the greenhouse. AM identified significant peaks in genomic regions of known SDS resistance quantitative trait loci cqSDSO01, c qRfs4,
\end{abstract}

Electronic supplementary material The online version of this article (doi:10.1007/s11032-015-0324-3) contains supplementary material, which is available to authorized users.

Y. Bao

Department of Agronomy and Plant Genetics, University of Minnesota, 411 Borlaug Hall, St. Paul,

MN 55108-6026, USA

e-mail: baoy@umn.edu

J. E. Kurle · G. Anderson · N. D. Young ( $ه)$

Department of Plant Pathology, University of Minnesota,

495 Borlaug Hall, St. Paul, MN 55108, USA

e-mail: neviny@umn.edu and SDS11-2. Additionally, two novel loci, one on chromosome 3 and another on chromosome 18, were tentatively identified. A ninefold cross-validation scheme was used to assess the prediction accuracy of GS for SDS resistance. The prediction accuracy of single-trait GS (ST-GS) was 0.64 for RLS, but less than 0.30 for RR, DMR, and FSS. Compared to STGS, none of multi-trait GS (MT-GS) models significantly improved the prediction accuracy due to weak correlations between the four traits. This study suggests both AM and GS hold promise for implementation in genetic improvement of SDS resistance in existing soybean breeding programs.

Keywords Association mapping - Early maturity · Genomic selection · Soybean · Sudden death syndrome

$\begin{array}{ll}\text { Abbreviations } \\ \text { AM } & \text { Association mapping } \\ \text { DMR } & \text { Dry matter reduction } \\ \text { FDR } & \text { False discovery rate } \\ \text { FSS } & \text { Foliar symptom severity } \\ \text { GEBV } & \text { Genomic estimated breeding value } \\ \text { GS } & \text { Genomic selection } \\ \text { LD } & \text { Linkage disequilibrium } \\ \text { MAF } & \text { Minor allele frequency } \\ \text { MAS } & \text { Marker-assisted selection } \\ \text { PCA } & \text { Principal component analysis } \\ \text { QTL } & \text { Quantitative trait loci }\end{array}$




$\begin{array}{ll}\text { REML } & \text { Restricted maximum likelihood } \\ \text { RLS } & \text { Root lesion severity } \\ \text { RR } & \text { Root retention } \\ \text { RR-BLUP } & \begin{array}{l}\text { Ridge-regression best linear unbiased } \\ \text { prediction }\end{array} \\ \text { SDS } & \text { Soybean sudden death syndrome } \\ \text { SNP } & \text { Single-nucleotide polymorphism }\end{array}$

\section{Introduction}

Sudden death syndrome (SDS) of soybean [Glycine $\max ($ L.) Merr.], caused by Fusarium virguliforme (Aoki et al. 2003), is an important disease that continues to spread across northern growing regions in the USA (Bernstein et al. 2007; Chilvers and Brown-Rytlewski 2010; Malvick and Bussey 2008; Navi and Yang 2008; Kurle et al. 2003), causing significant yield losses in infected fields (Wrather and Koenning 2009). Hyphae penetrate soybean roots and eventually colonize the vascular tissue of the plant causing the development of root rot (Jin et al. 1996). Subsequently, phytotoxin FvTox1 is produced by $F$. virguliforme and translocated to plant leaves during reproductive stages, causing diagnostic foliar symptoms such as leaf scorch (Brar et al. 2011; Jin et al. 1996). Both the root rot and leaf scorch lead to yield losses varying from 5 to $80 \%$ in individual soybean fields greatly affected by environmental conditions (Roy et al. 1997).

Crop rotation is generally ineffective in reducing the occurrence and severity of SDS because $F$. virguliforme in the form of chlamydospore or macroconidia can persist in crop residue and soil for many years (Roy et al. 1997). Therefore, SDS management relies heavily on planting resistant or tolerant cultivars complemented by optimal cultural practices. To date, soybean cultivars with partial resistance to SDS have been identified and developed (Hartman et al. 1997; Mueller et al. 2002, 2003; Njiti et al. 2002; Schmidt et al. 1999). However, no highly resistant soybean cultivars adapted to northern growing regions are yet available for soybean growers to use. Consequently, there is an urgent need to develop early maturing soybean cultivars with effective and durable resistance to SDS.

Developing SDS-resistant soybean cultivars has proven difficult mainly due to the complex genetic basis of SDS resistance, the interaction of pathogen and plant with the environment, and imperfect screening methods. Both the pathogen and disease are greatly influenced by environmental factors such as temperature, soil fertility, soil texture, rainfall, and planting date, which makes characterization and evaluation of cultivar performance extremely challenging (de Farias Neto et al. 2006; Gongora-Canul and Leandro 2011a, b; Jin et al. 1996; Sanogo and Yang 2001; Vick et al. 2003). For example, rainfall and temperature in the early season can lead to great variability in evaluation of SDS resistance in soybean genotypes because cool and wet conditions favor the initial infection of soybean roots by $F$. virguliforme, while weather during reproductive stages influences foliar symptom expression. In order to accurately screen for resistance to SDS, extensive field trials of soybean genotypes across multiple locations and years are necessary.

The genetic architecture of (partial) resistance to SDS is complex. A total of 58 QTL have been reported as providing resistance to SDS in bi-parental mapping populations (www.soybase.org, verified June 11, 2014), and only a few of them have been consistent across mapping populations from different genetic backgrounds (Kazi et al. 2008). However, the resistance loci $\mathrm{c} q R f s 4$ on linkage group C2 (chromosome 6), cqSDS001 on linkage group D2 (chromosome 17), cqRfs 1, cqRfs2, $c q R f s 3$ on linkage group $\mathrm{G}$ (chromosome 18), and $c q R f s 6$ on linkage group $\mathrm{N}$ (chromosome 3 ) were repeatedly mapped in multiple populations (de Farias Neto et al. 2007; Hnetkovsky et al. 1996; Iqbal et al. 2001; Kassem et al. 2006; Kazi et al. 2008; Lightfoot et al. 2001; Njiti et al. 1998, 2002; Prabhu et al. 1999). Potentially, the genetic markers identified from previous QTL mapping studies can assist in the selection of SDSresistant cultivars in a timely and resource-efficient manner (Prabhu et al. 1999). Luckew et al. (2013) recently evaluated ten confirmed SDS QTL in $\mathrm{F}_{2}$-derived lines from six populations and suggested the possibility of stacking QTL to achieve durable SDS resistance.

QTL mapping in bi-parental populations has been limited by the specific genetic backgrounds of the population under study, which reduces the ability to detect resistance genes. By contrast, association mapping (AM) (Rafalski 2002) provides an opportunity to identify QTL at a higher resolution by taking advantage of historical linkage disequilibrium (LD) in diverse populations. With increasing numbers of single-nucleotide polymorphisms (SNPs) combined with declining costs in genotyping, AM has become an attractive 
approach for revealing the genetic basis of target traits in crop species (Asoro et al. 2013; Bao et al. 2014; Huang et al. 2010; Mamidi et al. 2011, 2014; Jia et al. 2013; Sukumaran et al. 2012; Zhou and Steffenson 2013; Zhou et al. 2014). A recently published AM study identified QTL for SDS resistance in two soybean panels composed of released cultivars and advanced breeding lines from multiple soybean breeding programs (Wen et al. 2014). Compared to this earlier study, a panel of early maturing soybean lines adapted to short season growing condition was investigated and carefully evaluated below-ground phenotypes including root lesion severity and root retention in the greenhouse. The results suggest that SDS disease analysis based on root symptoms can be more informative with higher heritability than other approaches to SDS phenotyping.

A new marker-based approach known as genomic selection (GS) could potentially be an alternative strategy to stack numerous genes to achieve durable SDS resistance. Rather than utilizing only molecular markers in tight association with targeted QTLs, GS has been developed with the aim of directly predicting genetic value for quantitative traits by taking advantage of all available genome-wide marker information (Bernardo and Yu 2007; Meuwissen and Goddard 2010). In the GS scheme, QTL mapping is replaced by genomic prediction model training which involves fitting both phenotypic and genotypic data from a training population in either linear or nonlinear models. Marker effects estimated from the models are subsequently summed up to estimate genomic breeding values of individuals in a validation or breeding population with only genotypic data. Previous results in crop species, including soybean, have indicated that GS holds the potential to improve disease resistance with complex genetic architecture in breeding programs (Bao et al. 2014; Lorenz et al. 2012; Rutkoski et al. 2012, 2014). Here, we seek to investigate the potential use of GS to select SDS resistance in a typical public soybean breeding program with a focus on early maturing germplasm.

\section{Materials and methods}

Population, genotyping, population structure, and linkage disequilibrium

Details about the population and genotyping strategy were described previously, as were characterization of the population structure and linkage disequilibrium (LD) (Bao et al. 2014). Briefly, 282 soybean lines were selected including ancestral lines, advanced breeding lines, released public cultivars, and landraces from University of Minnesota Soybean Breeding Program (Table S1; Bao et al. 2014). An Illumina GoldenGate assay with 1536 SNP markers was used to genotype the selected soybean lines (Hyten et al. 2010). A total of 1247 SNP markers with greater than $5 \%$ minor allele frequency (MAF) and missing data rate less than $50 \%$ were used in subsequent analyses (Bao et al. 2014). Both STRUCTURE (Pritchard et al. 2000) and principal component analysis (PCA) identified a pattern of three clusters in the population approximately corresponding to three distinct genetic groups (Table S1; Bao et al. 2014). LD was characterized and illustrated using Haploview4.2 (Barrett et al. 2005).

Phenotyping and data analysis

In spring 2013, a total of 279 soybean lines (seeds of three lines were unavailable) were evaluated for SDS resistance in the greenhouse using the inoculation procedure of Luckew et al. (2012). An isolate of $F$. virguliforme, Somerset \#1A, originating in Minnesota had been maintained on PDA until it was used to inoculate autoclaved sorghum for use in these screening experiments. The sorghum was prepared for inoculation by soaking 1.5 liter quantities overnight in sterilizable spawn bags (Fungi Perfecti LLC, Olympia, WA) followed by autoclaving and cooling. The cooled sorghum was then inoculated with $15 \times 5 \mathrm{~mm}$ blocks of PDA infested with 2-week-old cultures of the Somerset \#1A isolate. Bags were incubated at room temperature with normal fluorescent room lighting for 30 days. The contents of each bag were mixed daily to ensure uniform infestation of the sorghum throughout the bag. At the time of soybean planting, the growth media was inoculated with a 1:20 (volume/volume) ratio of infested sorghum inoculum to media. The uninoculated control treatment contained only growth media. Each entry was planted in a Jumbo Junior (Belden Plastics Co., St. Paul, MN) square pot containing $800 \mathrm{ml}$ of soil. After planting, the pots were placed in the greenhouse, watered to field capacity daily, and maintained at $22{ }^{\circ} \mathrm{C}$ with 14 h daylight.

The greenhouse experiment was conducted as six separate plantings because of space limitations. The six plantings were conducted consecutively under the 
same greenhouse conditions. Each planting consisted of 30-50 soybean lines with five inoculated replications plus one uninoculated replication for each line. Two check cultivars: 'McCall' (susceptible) and 'MN0302' (resistant) were included in each planting. For each planting, each plant was evaluated for four symptoms or responses associated with SDS by the same experienced evaluator 4 weeks after planting. These observations included: root lesion severity (RLS), foliar symptom severity (FSS), root retention (RR), and dry matter reduction (DMR).

RLS is a measure of the severity of root lesion development caused by $F$. virguliforme infection ranging from 1 (no lesion) to 10 (most severe lesion development): $1=$ no lesions visible on taproot, $2=$ lesions on $10 \%$ of the taproot, $3=$ lesions on $20 \%$ of the taproot, $4=$ lesions on $30 \%$ of the taproot, $5=$ lesions on $40 \%$ of the taproot, $6=$ lesions on $50 \%$ of the taproot, $7=$ lesions on $60 \%$ of the taproot, $8=$ lesions on $70 \%$ of the taproot, $9=$ lesions on 90 to $100 \%$ of the taproot, and $10=$ lesions on $>90 \%$ of the taproot of the taproot or the taproot is completely missing.

FSS is a rating of the severity of leaf scorch caused by $F$. virguliforme (Bowen and Slaminko 2008, personal communication; Chawla et al. 2013): $1=$ no scorch, 2 = slight symptom development, with mottling on leaves, $3=$ moderate symptom development with interveinal chlorosis and necrosis, $4=$ intermediate symptom development with interveinal chlorosis and necrosis, $5=$ severe interveinal chlorosis and necrosis accompanied by cupping, $6=$ interveinal chlorosis and necrosis accompanied by cupping with some defoliation, $7=$ most leaves displaying necrosis, and $8=$ dead plants.

Percentage of root or shoot dry weight change caused by $F$. virguliforme infection was calculated as $\mathrm{RR}=$ (root dry weight of inoculated plant)/(root dry weight of uninoculated plant $) \times 100 . \mathrm{DMR}=100-$ (shoot dry weight of inoculated plant)/(shoot dry weight of uninoculated plant) $\times 100$.

The ratings of each trait were then fitted into a linear regression model: $y=u+L+\varepsilon$ within each planting and performed analysis of variation (ANOVA) with the PROC ANOVA in Statistical Analysis System (SAS) Version 9.4 (Cary, NC), where y was one of the four trait ratings of each plant, $u$ was the intercept, $L$ was the effect of soybean line, and $\varepsilon$ was the residual. The effect of line $\times$ replication was used as the error term to test significance of the effect of line. The phenotypic value of each soybean line was represented as the mean of trait ratings across five replications for each trait and used the phenotypic values for subsequent AM and GS modeling. Scatter plots were constructed based on the pair-wise correlation between the phenotypic values of each pair of traits.

\section{Association mapping}

We performed AM for RLS, FSS, RR, and DMR, respectively, with mixed linear model in the 'rrBLUP' package (Endelman 2011) in R (R Development Core Team 2005). The mixed linear model: $y=X \alpha+P \beta+K \gamma+\varepsilon$ was used, where $y$ is the vector of phenotypic values, $X$ is the vector of SNP marker genotypes, $\alpha$ is the coefficient of marker effect being estimated, $P$ is the matrix of first three principal components from PCA accounting for the population structure plus the covariate vector of experimental plantings, $\beta$ is the coefficient of principal components and experimental plantings, $K$ is the additive relationship matrix estimated based on SNP genotypes accounting for genetic kinship among the individuals, $\gamma$ is the vector of random effects corresponding to genetic kinship, and $\varepsilon$ is the vector of random effects corresponding to residuals. The variances of $\gamma$ and $\varepsilon$ are $\operatorname{Var}(\gamma)=2 K V_{\mathrm{g}}$ and $\operatorname{Var}(\varepsilon)=V_{\mathrm{R}}$, respectively, where $K$ is the genetic kinship, $V_{\mathrm{g}}$ is the genetic variance, and $V_{\mathrm{R}}$ is the residual variance. False discovery rate (FDR) of 0.05 was used to correct for multiple comparisons in AM using package 'QVALUE' in $\mathrm{R}$ ( $\mathrm{R}$ Development Core Team 2005). SNP markers with FDR $q$ value $<0.05$ were defined as significant SNPs associated with SDS resistance. Given the low SNP density on the genotyping panel, significant SNP markers are not expected to be exact locations of causal genes controlling variation of plant response to SDS. In the vicinity of the significant SNPs, previously described SDS resistance QTL was scanned in soybean genome (www. soybase.org). Manhattan plots were created based on the AM results with SNPEVG (Wang et al. 2012).

Prediction accuracy of genomic selection

To assess prediction accuracy of genomic selection (GS) for SDS resistance, the same set of phenotypic and genotypic data was used in a ninefold cross-validation study. Specifically, 279 soybean lines first were 
randomly divided into nine subsets. In each fold, eight subsets of lines (248 lines) were used as training sets and the remaining subset (31 lines) was a validation set. In the training set, the marker effects were simultaneously estimated by fitting a statistical model to both phenotypic and genotypic data. The marker effects were then used to predict the genetic values of individuals in the validation set. Prediction accuracy was calculated as the correlation between marker-based prediction and phenotypic values. The cross-validation process was repeated nine times (nine folds), with every subset of soybean lines used exactly once as the validation set.

\section{Genomic selection model}

Since there are four phenotypic traits associated with SDS resistance in the data set, both single-trait genomic selection (ST-GS) model and multi-trait genomic selection (MT-GS) were evaluated, and their accuracies for predicting SDS resistance were compared. For ST-GS, a mixed linear model was constructed to estimate marker effects of phenotypic traits: $y=X b+Z \alpha+e$, where $y$ is the vector $(n \times 1)$ of phenotypic observations of $n$ individuals, $X$ is the design matrix $(n \times r)$ for fixed planting effects, $b$ is the vector $(r \times 1)$ of planting effects, $Z$ is the design matrix $(n \times m)$ for additive effects of SNP markers, $\alpha$ is the vector $(m \times 1)$ of additive effects of SNP markers, and $e$ is the vector $(n \times 1)$ of residuals. The variances of $\alpha$ and $e$ are $\operatorname{Var}(\alpha)=I_{m} \sigma_{\alpha}^{2}$ and $\operatorname{Var}(e)=I_{n} \sigma_{e}^{2}$, respectively, where $I_{m}$ is the $m \times m$ identity matrix, $\sigma_{\alpha}^{2}$ is the additive genetic variance for each maker, $\sigma_{e}^{2}$ is the residual variance, and $I_{n}$ is the $n \times n$ identity matrix. A computationally efficient method, ridge-regression best linear unbiased prediction (RR-BLUP) was employed to solve the mixed model. Previous GS studies suggested slight differences between different genomic prediction algorithms including G-BLUP (which is equivalent to RRBLUP), Bayesian approaches, and machine learning algorithms (Asoro et al. 2011; Bao et al. 2014; Lorenzana and Bernardo 2009; Lorenz et al. 2012; Rutkoski et al. 2012). The marker effects were simultaneously estimated by solving the mixed model through the restricted maximum likelihood (REML) method implemented in $\mathrm{R}$ package 'rrBLUP' (R Development Core Team 2005). Variance of additive effects and variance of residual effects were estimated.
MT-GS models were developed by fitting the phenotypic observations of multiple traits $(\mathrm{t})$ simultaneously in a multivariate mixed linear model: $y=\left(I_{t} \otimes X\right) b+\left(I_{t} \otimes Z\right) \alpha+e$, where $y$ is the matrix $(n \times t)$ of phenotypic observations for $\mathrm{t}$ traits of $\mathrm{n}$ individuals, $I_{t}$ is the identity matrix $(t \times t), X$ is the design matrix $(n \times r)$ for fixed planting effects for each trait, $b$ is the matrix $(r \times t)$ of planting effects for $\mathrm{t}$ trait, $Z$ is the design matrix $(n \times m)$ for additive effects of SNP markers for each trait, $\alpha$ is the matrix $(m \times t)$ of additive effects of SNP markers for $t$ trait, $e$ is the matrix $(n \times t)$ of residuals, and $\otimes$ denotes the Kronecker product. The variances of $\alpha$ and $e$ are $\operatorname{Var}(\alpha)=G_{0} \otimes A$ and $\operatorname{Var}(e)=R_{0} \otimes I_{n}$, respectively, where $G_{0}$ is the covariance matrix $(t \times t)$ of additive effects, $\mathrm{A}$ is the additive genetic relationship matrix $(n \times n), R_{0}$ is the covariance matrix $(t \times t)$ of residuals, and $I_{n}$ is the identity matrix $(n \times n)$. The marker effects of each trait were simultaneously estimated by solving the mixed model through REML method implemented in $\mathrm{R}$ package 'rrBLUP' ( $\mathrm{R}$ Development Core Team 2005). The pair-wise genetic correlation was estimated as $\sigma_{g 12} \sqrt{\sigma_{g 11} \sigma_{g 22}}$, where $\sigma_{g}$ is the genetic variance-covariance matrix for multiple traits. $\sigma_{g}$ was calculated as $\sum_{i=1}^{m} \operatorname{Var}\left(\mathrm{SNP}_{i}\right) \alpha_{i} \alpha_{i}^{T}$ (Jia and Jannink 2012), where $\operatorname{var}\left(\mathrm{SNP}_{i}\right)$ is the genotype variance for $\mathrm{SNP}_{i}$ and $\alpha$ is the vector $(\mathrm{m} \times 1)$ of additive effects for $\mathrm{SNP}_{i}$. The additive genetic variance and the residual variance were estimated. Ten types of MT-GS models were developed: RLS_FSS model for RLS and FSS; RLS_RR model for RLS and RR; RLS_DMR model for RLS and DMR; FSS_RR model for FSS and RR; FSS_DMR model for FSS and DMR; RR_DMR model for RR and DMR; RLS_FSS_DMR model for RLS, FSS, and DMR; RLS_FSS_RR model for RLS, FSS, and RR; RR_FSS_DMR model for RR, FSS, and DMR; and FT model for all four traits. A notched boxplot was made to compare the prediction performance of MTGS models to ST-GS models for each trait. The notch marks the $95 \%$ confidence interval for the medians. In the notched boxplot, the medians significantly differ if two boxes' notches do not overlap.

\section{Marker number}

The effect of marker numbers on GS accuracy was also determined through nine-fold cross-validation by 
including random samples of 96, 192, 384, and 768 SNPs from the full marker set. Within each fold, the analysis was repeated 100 times to avoid sampling bias for markers. All prediction accuracies were estimated with R package 'rrBLUP' (R Development Core Team 2005). A notched boxplot was made to compare the prediction performance of GS models with different subsets of markers for each trait. The notch marks the $95 \%$ confidence interval for the medians. In the notched boxplot, the medians significantly differ if two boxes' notches do not overlap.

\section{Results}

Phenotypic data

Analysis of variance (ANOVA) for each of four SDS resistance traits was conducted within each planting. ANOVA showed the effect of soybean genotypes was significant $(p<0.05)$ in all plantings, except for FSS in plantings 4 and 6 , and RR and DMR in planting 5 (Table 1). The lack of significance of genotype effect in ANOVA indicated that the effect of replication $\times$ genotype contributed a large portion of the trait variation within the planting.

Susceptible and resistant check cultivars were set up to provide the means of comparing phenotyping performance in the six plantings. As expected, the susceptible check 'McCall' exhibited high RLS scores ranging from 5.5 to 8.8 within plantings with an exception of 2.4 in Planting 3; the resistant check 'MN0302' exhibited low RLS scores ranging from 2.2 to 4.6 within plantings with an exception of 6.4 in Planting 1 (Data not shown).

In general, soybean lines showed a wider range of responses to SDS for both RR and DMR than RLS and FSS scores (Supplemental Fig. S1). The phenotypic data density of RLS was more evenly distributed than that of the other three traits (Supplemental Fig. S1). RLS scores ranged from 2.4 to 10 with a total of 49 lines exhibiting scores less severe than the resistant check 'MN0302' (Supplemental Fig. S1). FSS scores ranged from 1 to 8 with a total of 81 lines that did not develop any foliar symptoms plus another 43 less severe than 'MN0302' (Supplemental Fig. S1). The range observed in RR was $0-1141 \%$ with a total of 69 lines more resistant than 'MN0302' (Supplemental Fig. S1). A total of 29 lines did not show any dry matter reduction plus another 64 lines with DMR less severe than 'MN0302' (Supplemental Fig. S1). Based on all four traits associated with SDS resistance, 11 soybean lines consistently exhibited symptoms less severe than that of the resistant check 'MN0302' and have potential to be used as breeding parents in the SDS resistance improvement program (Supplemental Table S1).

Pair-wise correlations of traits

The pair-wise correlations between the phenotypic values of each pair of traits were calculated and are displayed in scatter plots (Fig. 1). As expected, a strong negative correlation was observed between RR and DMR, while RLS and FSS were positively correlated with $r=0.47$ (Fig. 1). By contrast, the correlations between RR and RLS, DMR and FSS, RLS and FSS, and RR and FLS were poor (Fig. 1). Similar pair-wise phenotypic correlations within each of six plantings (Data not shown) were observed, and pair-wise genetic correlation of traits was consistent with the observation in phenotypic correlation (Supplemental Table S2).

Significant markers in association mapping analysis

Association mapping (AM) was performed for RLS, FSS, RR, and DMR. The QQ plot indicated the model we implemented for AM was sufficient to control the false positive (Supplemental Fig. S2). We identified two and eight significant (qFDR $<0.05$ ) SNP markers for DMR and RR, respectively, but none for the other two traits (Table 2; Supplemental Fig. S3). Among the eight distinct significant markers, three were in the same genomic intervals as the known SDS resistance quantitative trait loci (QTL) cqSDSO01 on linkage group D2 (chromosome 17) (Table 2; Supplemental Fig. S3). Another marker at position $80.28 \mathrm{cM}$ on linkage group $\mathrm{C} 2$ (chromosome 6) was in the genomic region of cqRfs4 (Table 2; Supplemental Fig. S3). Both $c q S D S 001$ and $c q R f s 4$ have been previously identified and confirmed in multiple bi-parental populations (de Farias Neto et al. 2007; Hnetkovsky et al. 1996; Iqbal et al. 2001; Kassem et al. 2012; Kazi et al. 2008; Njiti et al. 2002). Additionally, two significant SNP markers in the present study confirmed a previously identified QTL, SDS11-2, on 
Table 1 ANOVA for four SDS resistance traits within each planting

$R L S$ root lesion severity,

FSS foliar symptom severity, $R R$ root retention, $D M R$ dry matter reduction, $D f$ degree of freedom, $M S$ mean of square

* $P \leq 0.05$; ** $P \leq 0.01$; *** $\bar{P} \leq 0.001$

Fig. 1 Scatter plots of pairwise correlation of traits associated with SDS resistance. $R L S$ root lesion severity, FSS foliar symptom severity, $R R$ root retention (\%), DMR dry matter reduction (\%). The values in the scatter plot matrix represent the $r$ values of pair-wise correlation of traits

\begin{tabular}{|c|c|c|c|c|c|c|c|c|c|}
\hline \multirow[t]{2}{*}{ Planting } & \multirow[t]{2}{*}{ Source } & \multicolumn{2}{|c|}{ RLS } & \multicolumn{2}{|l|}{ FSS } & \multicolumn{2}{|l|}{ RR } & \multicolumn{2}{|c|}{ DMR } \\
\hline & & Df & MS & Df & MS & Df & MS & Df & MS \\
\hline \multirow[t]{2}{*}{1} & Line & 50 & $7.20 * *$ & 50 & $11.55^{* *}$ & 49 & $0.68 * *$ & 49 & $0.33 * *$ \\
\hline & Error & 198 & 3.82 & 198 & 6.28 & 194 & 0.36 & 194 & 0.20 \\
\hline \multirow[t]{2}{*}{2} & Line & 51 & $7.44 * * *$ & 51 & $5.66^{* *}$ & 51 & $0.28 * *$ & 51 & $0.19 * * *$ \\
\hline & Error & 184 & 3.37 & 184 & 3.61 & 184 & 0.15 & 184 & 0.07 \\
\hline \multirow[t]{2}{*}{3} & Line & 46 & $11.78 * * *$ & 46 & $7.08 * * *$ & 46 & $0.62 * *$ & 46 & $2.38 * * *$ \\
\hline & Error & 188 & 4.66 & 188 & 3.53 & 188 & 0.36 & 188 & 0.16 \\
\hline \multirow[t]{2}{*}{4} & Line & 50 & $9.62 *$ & 50 & 6.41 & 50 & $1.13 * *$ & 50 & $2.98 * * *$ \\
\hline & Error & 191 & 7.85 & 191 & 5.53 & 191 & 0.66 & 191 & 1.03 \\
\hline \multirow[t]{2}{*}{5} & Line & 49 & $9.65 * * *$ & 49 & $10.20 * *$ & 49 & 0.15 & 49 & 0.09 \\
\hline & Error & 177 & 4.38 & 177 & 5.63 & 176 & 0.13 & 177 & 0.08 \\
\hline \multirow[t]{2}{*}{6} & Line & 31 & $12.73 * *$ & 31 & 4.19 & 31 & $18.97 * * *$ & 31 & $15.22 * * *$ \\
\hline & Error & 111 & 7.22 & 111 & 3.99 & 111 & 1.75 & 111 & 1.28 \\
\hline
\end{tabular}

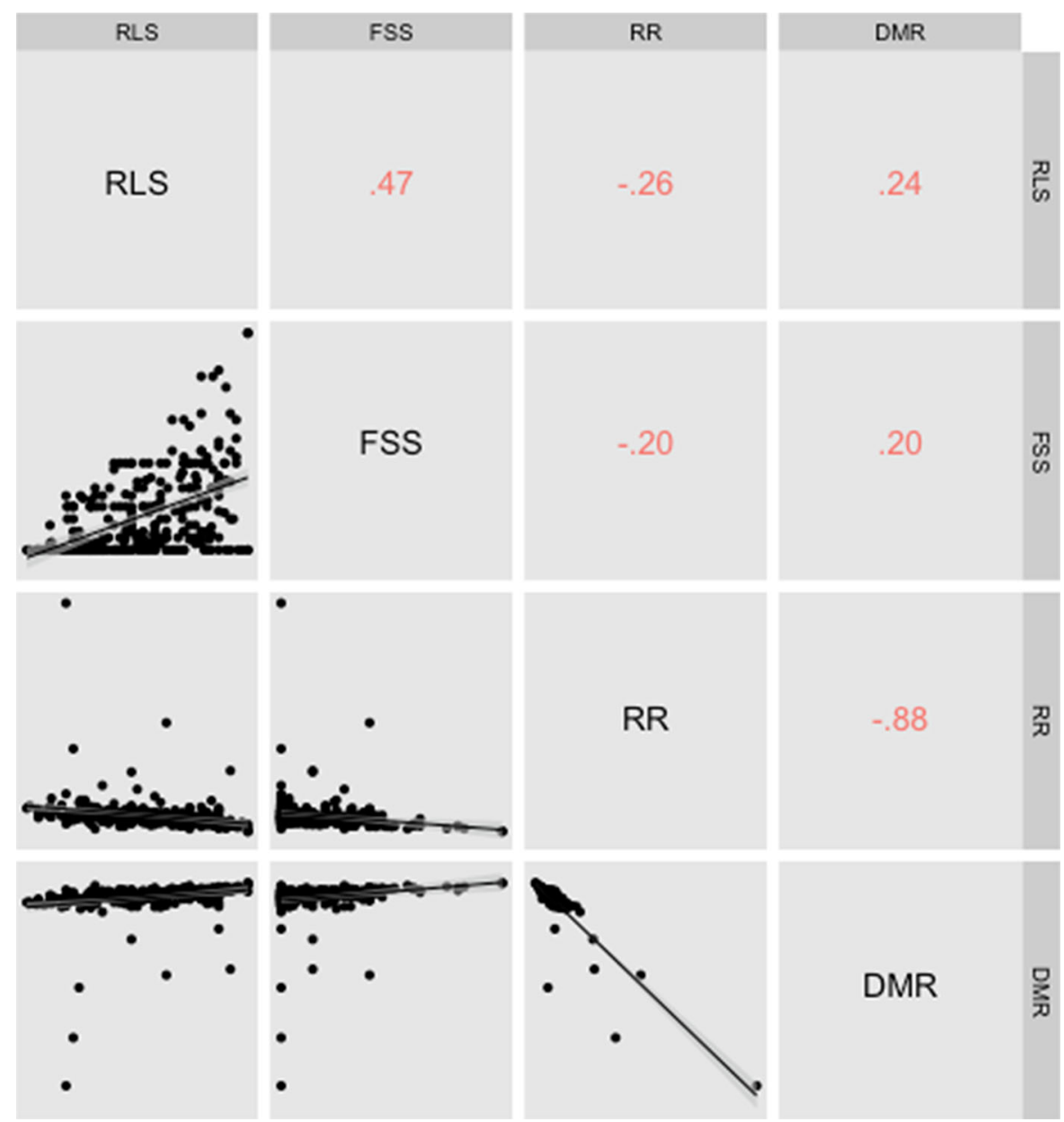


Table 2 The significant SNPs (false discovery rate <0.05) detected from association mapping (AM) for SDS resistance

\begin{tabular}{llllllll}
\hline Trait & Marker & LG & Chromosome & Position (cM) & Position (bp) & $P$ & qFDR \\
\hline RR & BARC-044643-08744 & N & 3 & 4.71 & 460,387 & $2 \mathrm{E}-04$ & 0.03 \\
& BARC-028177-05786 & C2 & 6 & 80.28 & $13,550,856$ & $1 \mathrm{E}-04$ & 0.02 \\
& BARC-051665-11191 & D2 & 17 & 72.14 & $14,849,926$ & $2 \mathrm{E}-07$ & 0.0002 \\
& BARC-023721-03465 & D2 & 17 & 75.11 & $20,352,435$ & $2 \mathrm{E}-04$ & 0.03 \\
& BARC-064101-18557 & D2 & 17 & 75.44 & $25,852,278$ & $2 \mathrm{E}-05$ & 0.008 \\
& BARC-059487-15840 & D2 & 17 & 76.12 & $35,057,016$ & $1 \mathrm{E}-05$ & 0.006 \\
& BARC-061049-17016 & D2 & 17 & 77.39 & $36,090,548$ & $7 \mathrm{E}-06$ & 0.005 \\
& BARC-024251-04812 & G & 18 & 94.3 & $59,472,567$ & $6 \mathrm{E}-05$ & 0.002 \\
DMR & BARC-051665-11191 & D2 & 17 & 72.14 & $14,849,926$ & $2 \mathrm{E}-05$ & 0.01 \\
& BARC-024251-04812 & G & 18 & 94.3 & $59,472,567$ & $6 \mathrm{E}-06$ & 0.008 \\
\hline
\end{tabular}

$R R$ root retention, $D M R$ dry matter retention, $L G$ linkage group

a $q F D R q$ value of false discovery rate (FDR) estimated with R package "QVALUE." SNP markers with FDR $q$ value $<0.05$ were defined as significant SNPs associated with SDS resistance

linkage group D2 (chromosome 17) (Kazi et al. 2008). The rediscovery of the previously identified QTL strengthened the confidence in the overall quality of AM analysis. Notably, a SNP marker near the telomere on chromosome 3 (linkage group $\mathrm{N}$ ) was tentatively identified as associated with $\mathrm{RR}$ variation, and another SNP marker near the telomere on chromosome 18 (linkage group G) was associated with the variation in both RR and DMR (Table 2; Supplemental Fig. S3; Supplemental Fig. S4). Since no previous QTL had been discovered near these two genomic locations, the two newly identified loci were provisionally named SDS14-1 (on chromosome 3) and SDS14-2 (on chromosome 18). For each of the five loci (namely, cqSDS001, cqRfs4, SDS11-2, SDS14-1, and SDS14-2), RR and DMR peaks were coincident with each other (Supplemental Fig. S4).

Single-trait versus multi-trait genomic selection

Besides identifying causal loci associated with SDS resistance through $\mathrm{AM}$, the phenotypic and genotypic data sets were used to evaluate the utility of GS in predicting SDS resistance phenotypes. Overall, the prediction accuracy of the ST models was 0.64, 0.20, 0.18 , and 0.16 for RLS, FSS, RR, and DMR, respectively (Fig. 2). However, among these ST models, only the prediction accuracy for RLS was significantly different from zero. To determine whether multi-trait (MT) models improved prediction accuracy, ST and MT models were also compared. Compared to ST models, none of MT-GS models significantly improved the prediction accuracy for any trait (Fig. 2). The RLS_FSS_DMR model did increase the prediction accuracy for DMR from 0.16 to 0.25 while maintaining a similar accuracy for FSS, but reduced the accuracy for RLS to 0.26 (Fig. 2). The FT model performed equivalently to ST models for all four traits (Fig. 2).

\section{Marker number effect on prediction accuracy}

To determine the effect of marker number on prediction accuracy, the prediction accuracy with different sizes of marker set used in ST-GS models was compared. The prediction accuracy generally increased as the number of SNP markers increased for RLS, RR, and DMR, but not for FSS (Fig. 3). The rate of gain in accuracy was greatest when the marker set increased from 96 to 192 (Fig. 3). With 96 random genome-wide SNPs, the prediction accuracy of ST model was only $0.25,0.02,0.14$, and 0.04 for RLS, FSS, RR, and DMR, respectively (Fig. 3).

\section{Discussion}

Accurate assessment of phenotypic variation is essential for understanding disease biology, effective resistance breeding, and dissection of genetic architecture. 


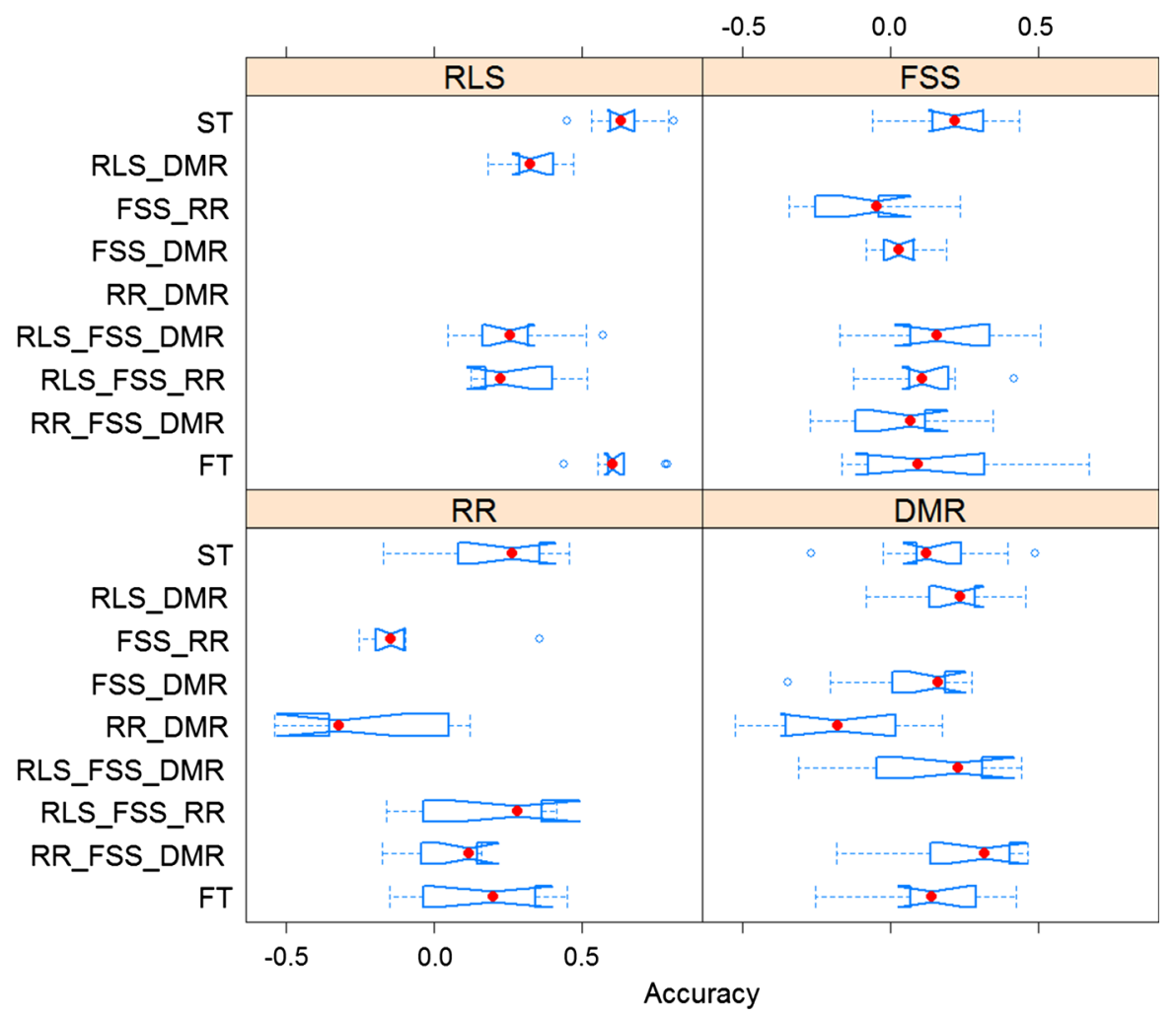

Fig. 2 Prediction accuracy with multi-trait genomic selection (GS) models compared with single-trait GS models for four SDS resistance traits. $R L S$ root lesion severity, $R R$ root retention, $F S S$ foliar symptom severity, $D M R$ dry matter reduction, $S T$ singletrait model, RLS_FSS model for RLS and FSS; RLS_RR model for RLS and RR; RLS_DMR model for RLS and DMR; FSS_RR model for FSS and RR; FSS_DMR model for FSS and

The heritability of greenhouse evaluation of SDS resistance ranged from 33 to $66 \%$ in previous studies (Njiti et al. 2001). In the greenhouse experiment, the effect of soybean genotypes was significant $(p<0.05)$ in most plantings indicating an overall reliability of phenotypic data (Table 1). However, we still observed substantial replication $\times$ genotype variation in four trait $\times$ planting experiments (Table 1). The high level of phenotypic variation between replications has also been observed in previous studies (Kazi et al. 2008; Luckew et al. 2013) and could be attributed to the complex genetic basis of SDS resistance, interactive effects of genotype with environment, and/or imperfect screening methods. Another limitation in the current study was the low throughput capacity of the phenotyping system; soybean lines had to be evaluated in six plantings, which might have reduced the ability
DMR; RR_DMR model for RR and DMR; RLS_FSS_DMR model for RLS, FSS, and DMR; RLS_FSS_RR model for RLS, FSS, and RR; RR_FSS_DMR model for RR, FSS, and DMR; and FT model for all four traits. Red dot represents median of accuracies for each model. Notch marks the $95 \%$ confidence interval for the medians. (Color figure online)

to detect all causative QTL and/or led to biased estimations. In other words, the genetic effects might have been confounded by the effect of consecutive experimental plantings conducted over time, limiting the ability to induce SDS symptoms consistently, and as a result, reducing the explanatory power of AM. For example, changing light intensity and ambient temperature variation associated with seasonal changes in sun angle and ambient temperature presumably added to variability in SDS phenotype. To minimize the influences of these sources of variance of among plantings, we conducted the greenhouse experiments with supplemental lighting and air conditioning, and accounted for the effect of plantings as a fixed effect in the AM model.

Eight and two SNP markers in significant association with RR and DMR were identified, respectively, 
Fig. 3 Prediction accuracy with different numbers of markers for four SDS resistance traits. $R L S$ root lesion severity, $R R$ root retention, FSS foliar symptom severity, DMR dry matter reduction. Red dot represents median of accuracies for each subset of markers. Notch marks the $95 \%$ confidence interval for the medians. (Color figure online)

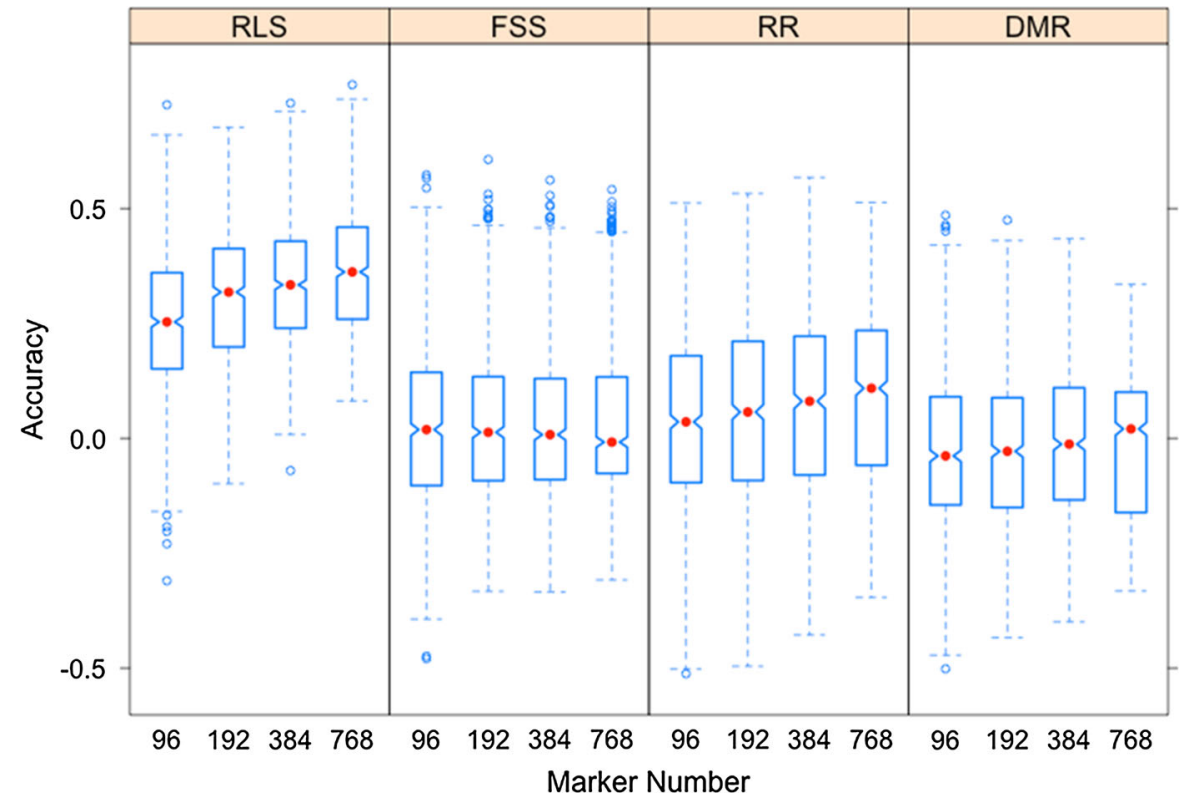

indicating a total of five loci underlying SDS resistance. Among the five loci identified in this study, cqSDSO01 and $c q R f s 4$ had been previously identified and confirmed in more than one population, which strengthens the confidence of the overall analysis. The cqSDS001 locus was first discovered at positions 78 and $85 \mathrm{cM}$ on linkage group D2 from the resistant sources PI567374 and Ripley, respectively (de Farias Neto et al. 2007) and was later confirmed in another population derived from Hartwig (Kazi et al. 2008). A second SDS resistance locus, $c q R f s 4$, was reported to be associated with foliar resistance (Kazi et al. 2008; Luckew et al. 2013; Triwitayakorn et al. 2005); however, we identified a significant SNP marker, BARC-028177-05786, underlying variation of RR in this QTL interval. Given increasing numbers of SNPs in newly developed genotyping assay for soybean (Song et al. 2013), higher resolution of genetic mapping might pinpoint the potential candidate genes in the genomic regions underlying SDS resistance. Additionally, two SNP markers on linkage group D2 were detected as being significantly associated with RR in our study, which adds support to the SDS11-2 locus identified previously in Kazi et al. (2008).

A cluster of SDS resistance genes, $c q R f s 1, c q R f s 2$, $c q R f s 3$, has been repeatedly mapped on linkage group G (chromosome 18) (Chang et al. 1996; Iqbal et al. 2001; Meksem et al. 1999; Njiti et al. 1998, 2002;
Prabhu et al. 1999; Kazi et al. 2008), but these genes were not detected in our collection of soybean accessions. More recently, Wen et al. (2014) detected a strong peak at or near the $R f s 2$ locus in all the SDS disease assessment criteria evaluated their AM study. Possible explanations for these differences include different sources of germplasm used in different studies as well as the methods used for resistance evaluation. In the present study, the mapping panel germplasm included released cultivars and advanced breeding lines adapted to Minnesota across maturity group 00 to $\mathrm{I}$ in contrast to the later maturing germplasm in Wen et al. (2014). In the present study, we also inoculated plants with an isolate of $F$. virguliforme originating in Minnesota. Finally, we extended the detailed examination to target belowground phenotypes in greenhouse. By contrast, SDS resistance was evaluated in naturally infested fields for above-ground symptoms in Wen et al. (2014). Since both root rot and leaf scorch traits are responsible for yield losses caused by SDS, the loci associated with below-ground phenotypes discovered in the present study should complement to the findings in Wen et al. (2014) and other earlier genetic mapping studies of SDS resistance.

Instead of $R f s 2$, a significant SNP marker BARC024251-04812 on the opposite end of chromosome 18 was identified, which accounted for variation in both 
$\mathrm{RR}$ and DMR. This SNP was about 1.7 Mb away from a previously described resistance QTL SDS4-2 (Njiti et al. 1998). Another novel locus was tagged by a SNP marker BARC-044643-08744 located near the telomeric region of chromosome 3 . These two novel loci could be validated in future investigation of either a biparental mapping population or another AM population with a higher density of SNP markers.

To pyramid these resistance QTL into commercial soybean cultivars, the significant SNP markers identified in present study can be developed as a breederfriendly SNP array for conducting MAS in SDS resistance breeding programs. However, stacking multiple QTL and introgressing them to an adapted elite parent would require considerable resources and time. As an alternative to stacking major SDS resistance genes, GS may provide breeders an opportunity to integrate a broader set of causative loci underlying SDS resistance with the goal of more durable resistant soybean cultivars. Despite successful rediscovery of known QTL for RR and DMR, we failed to identify any significant signals (qFDR $<0.05$ ) for RLS and FSS with AM. This might indicate that the genetic variation of RLS and FSS captured in the population is associated with numerous causative genes each with a small effect. In this case, genome-wide selection as implemented through GS is expected to be more effective than MAS because GS would enable breeders to select candidate lines with higher levels of cumulative resistance to SDS conferred by numerous small effect loci. Estimates of prediction accuracy for RLS were as high as 0.64 (Fig. 2), which is comparable to that for SCN resistance in soybean (Bao et al. 2014), Fusarium head blight resistance in barley and wheat (Lorenz et al. 2012; Rutkoski et al. 2012), and northern leaf blight in corn (Technow et al. 2013). Given the high prediction accuracy, GS holds great potential for implementation in genetic evaluation of breeding candidates in an actual soybean improvement program targeting at SDS resistance.

SDS resistance breeding is further complicated by the existence of two apparently distinct resistance mechanisms involved in expression of root versus foliar responses to SDS (Kazi et al. 2008; Triwitayakorn et al. 2005). Some known QTL confer specific resistance to root rot or foliar scorch, while others confer resistance to both (de Farias Neto et al. 2007; Hnetkovsky et al. 1996; Iqbal et al. 2001; Kassem et al. 2006; Kazi et al. 2008; Njiti et al. 1998, 2002). To develop soybean cultivars with both root and foliar resistance to SDS, multi-trait GS (MT-GS) has the potential to be an effective selection strategy for implementing an SDS resistance improvement program. An MT-GS model is developed by simultaneously fitting phenotypic data from the evaluations of root and foliar symptoms as dependent variables in the model. Subsequently, the MT-GS model using one marker panel leads to simultaneous prediction of both root and foliar symptoms.

Our results suggested that the prediction accuracy of GS model based on single traits (ST-GS) for FSS, RR, and DMR was comparatively low $(<0.3)$ (Fig. 2). In a simulation study, Jia and Jannink (2012) indicated that the prediction accuracy for low-heritability traits could be improved by GS models based on multiple related traits (MT-GS) models. The underlying mechanism of improved accuracy for low-heritability traits in MT-GS is presumably genetic relationship between the highly related traits (Jia and Jannink 2012). In the case of SDS resistance, we hypothesized that MT-GS might be capable of taking advantage of the genetic relationship between low-heritability traits: FSS, RR and DMR, and high-heritability trait: RLS. However, the FT model based on all four SDS resistance traits performed equivalently to the ST models in the study (Fig. 2) and none of the MT-GS models significantly improved the prediction accuracy. An increase in the prediction accuracy for DMR with the RLS_FSS_DMR model was observed, while the RLS_FSS_DMR model failed to maintain similar prediction accuracy for RLS and FSS as that in ST-GS models (Fig. 2).

A simulation study indicated that MT-GS greatly increased the prediction accuracy only when the genetic correlation between two related traits was higher than 0.7 (Jia and Jannink 2012). The MT-GS models performed equivalent to the ST-GS models; this indicates that the genetic basis of FSS, RR, and DMR might not be highly correlated with that of RLS. Indeed, consistently weak pair-wise correlation of FSS $\times$ RLS, RR $\times$ RLS and DMR $\times$ RLS was observed as shown in Fig. 1 and Table S2. Mueller et al. (2002) also suggested that the correlation between root rot and foliar severity was not significant. Considering that root rot is caused by direct infection of $F$. virguliforme (Jin et al. 1996), while foliar scorch is caused by phytotoxin FvTox1 produced by $F$. virguliforme (Brar et al. 2011; Jin et al. 1996), different 
genetic mechanisms appear to be involved in root versus foliar resistances.

\section{Conclusion}

The present study suggests AM could be used as an alternative method for mapping QTL underlying SDS resistance, and GS holds potential for implementation in genetic evaluation of root lesion severity associated with SDS. We conclude that SDS resistance is a complex disease trait, leading to numerous challenges in evaluating and breeding for SDS-resistant soybean cultivars. Firstly, improving phenotypic screening methods to ensure high-quality and high-throughput evaluation of SDS resistance should remain as an important component of the current SDS breeding program. Secondly, high-density genome-wide markers or sequence-based genotyping methods could be employed to dissect the genetic architecture of SDS resistance more precisely. Lastly, the realized response and cost-effectiveness of GS deserves further investigation in both greenhouse and field prior to implementing GS for developing durable SDS resistance in soybeans.

Acknowledgments We thank Colin Zumwalde, John Lencowski, Erin Walch, Adam Barbeau, Dante Leyva, Marissa Scherven, and other members of Soybean Pathology Lab at University of Minnesota for conducting the greenhouse experiments and disease resistance evaluation in the laboratory. The manuscript was also benefited through discussions with Dr. Rex Bernardo. This research was supported by Minnesota Agricultural Experiment Station project MN-22-015 and by Minnesota Soybean Production and Promotion Council grants 14-12C, 18-13CN and 5-14C.

Open Access This article is distributed under the terms of the Creative Commons Attribution 4.0 International License (http:// creativecommons.org/licenses/by/4.0/), which permits unrestricted use, distribution, and reproduction in any medium, provided you give appropriate credit to the original author(s) and the source, provide a link to the Creative Commons license, and indicate if changes were made.

\section{References}

Aoki T, O’Donnell K, Homma Y, Lattanzi AR (2003) Suddendeath syndrome of soybean is caused by two morphologically and phylogenetically distinct species within the Fusarium solani species complex- $F$. virguliforme in North America and F. tucumaniae in South America. Mycologia 95:660-684
Asoro FG, Newell MA, Beavis WD, Scott MP, Jannink J (2011) Accuracy and training population design for genomic selection on quantitative traits in elite North American oats. Plant Genome 4:132-144

Asoro FG, Newell MA, Scott MP, Beavis WD, Jannink J (2013) Genome-wide association study for beta-glucan concentration in elite North American oat. Crop Sci 53:542-553

Bao Y, Vuong T, Meinhardt C, Tiffin P, Denny R, Chen S, Nguyen HT, Orf JH, Young ND (2014) Potential of association mapping and genomic selection to explore PI88788 derived soybean cyst nematode resistance. Plant Genome. doi:10.3835/plantgenome2013.11.0039

Barrett JC, Fry B, Maller J, Daly M (2005) Haploview: analysis and visualization of LD and haplotype maps. Bioinformatics 21:263-265

Bernardo R, Yu J (2007) Prospects for genomewide selection for quantitative traits in maize. Crop Sci 47:1082-1090

Bernstein E, Atallah Z, Koval N, Hudelson B, Grau C (2007) First report of sudden death syndrome of soybean in Wisconsin. Plant Dis 91:1201

Brar HK, Swaminathan S, Bhattacharyya MK (2011) The Fusarium virguliforme toxin FvTox1 causes foliar sudden death syndrome-like symptoms in soybean. Mol Plant Microbe Interact 24:1179-1188

Chang S, Doubler T, Kilo V, Suttner R, Klein J, Schmidt M, Gibson P, Lightfoot D (1996) Two additional loci underlying durable field resistance to soybean sudden death syndrome (SDS). Crop Sci 36:1684-1688

Chawla S, Bowen CR, Slaminko TL, Hobbs HA, Hartman GL (2013) A public program to evaluate commercial soybean cultivars for pathogen and pest resistance. Plant Dis 97:568-578

Chilvers M, Brown-Rytlewski D (2010) First report and confirmed distribution of soybean sudden death syndrome caused by Fusarium virguliforme in Southern Michigan. Plant Dis 94:1164

de Farias Neto AL, Hartman GL, Pedersen WL, Li S, Bollero GA, Diers BW (2006) Irrigation and inoculation treatments that increase the severity of soybean sudden death syndrome in the field. Crop Sci 46:2547-2554

de Farias Neto AL, Hashmi R, Schmidt M, Carlson SR, Hartman GL, Li S, Nelson RL, Diers BW (2007) Mapping and confirmation of a new sudden death syndrome resistance QTL on linkage group D2 from the soybean genotypes PI 567374 and 'Ripley'. Mol Breed 20:53-62

Endelman JB (2011) Ridge regression and other kernels for genomic selection with R package rrBLUP. Plant Genome 4:250-255

Gongora-Canul C, Leandro L (2011a) Effect of soil temperature and plant age at time of inoculation on progress of root rot and foliar symptoms of soybean sudden death syndrome. Plant Dis 95:436-440

Gongora-Canul C, Leandro L (2011b) Plant age affects root infection and development of foliar symptoms of soybean sudden death syndrome. Plant Dis 95:242-247

Hartman G, Huang Y, Nelson R, Noel G (1997) Germplasm evaluation of Glycine max for resistance to Fusarium solani, the causal organism of sudden death syndrome. Plant Dis 81:515-518

Hnetkovsky N, Chang S, Doubler T, Gibson P, Lightfott D (1996) Genetic mapping of loci underlying field resistance 
to soybean sudden death syndrome (SDS). Crop Sci 36:393-400

Huang X, Wei X, Sang T, Zhao Q, Feng Q, Zhao Y, Li C, Zhu C, Lu T, Zhang Z (2010) Genome-wide association studies of 14 agronomic traits in rice landraces. Nat Genet 42:961-967

Hyten DL, Choi I, Song Q, Specht JE, Carter TE, Shoemaker RC, Hwang E, Matukumalli LK, Cregan PB (2010) A high density integrated genetic linkage map of soybean and the development of a 1536 universal soy linkage panel for quantitative trait locus mapping. Crop Sci 50:960-968

Iqbal M, Meksem K, Njiti V, Kassem MA, Lightfoot D (2001) Microsatellite markers identify three additional quantitative trait loci for resistance to soybean sudden-death syndrome (SDS) in Essex $\times$ Forrest RILs. Theor Appl Genet 102:187-192

Jia Y, Jannink J (2012) Multiple-trait genomic selection methods increase genetic value prediction accuracy. Genetics 192:1513-1522

Jia G, Huang X, Zhi H, Zhao Y, Zhao Q, Li W, Chai Y, Yang L, Liu K, Lu H (2013) A haplotype map of genomic variations and genome-wide association studies of agronomic traits in foxtail millet (Setaria italica). Nat Genet 45:957-961. doi:10.1038/ng.2673

Jin H, Hartman G, Nickell C, Widholm J (1996) Characterization and purification of a phytotoxin produced by Fusarium solani, the causal agent of soybean sudden death syndrome. Phytopathology 86:277-282

Kassem M, Shultz J, Meksem K, Cho Y, Wood A, Iqbal M, Lightfoot D (2006) An updated 'Essex'by 'Forrest'linkage map and first composite interval map of QTL underlying six soybean traits. Theor Appl Genet 113:1015-1026

Kassem MA, Ramos L, Leandro L, Mbofung G, Hyten DA, Kantartzi SK, Grier RL, Cianzio S, Meksem K (2012) The First 'PI438489B' by 'Hamilton' SNP-based genetic linkage map of soybean [Glycine $\max (\mathrm{L}$.) Merr.] and quantitative trait loci (QTL) that underlie SDS resistance. J Plant Genome Sci 1(2):18-30

Kazi S, Shultz J, Afzal J, Johnson J, Njiti V, Lightfoot DA (2008) Separate loci underlie resistance to root infection and leaf scorch during soybean sudden death syndrome. Theor Appl Genet 116:967-977

Kurle J, Gould S, Lewandowski S, Li S, Yang X (2003) First report of sudden death syndrome (Fusarium solani $f$. sp. glycines) of soybean in Minnesota. Plant Dis 87:449

Lightfoot DA, Gibson PT, Merkem K (2001) Soybean sudden death syndrome resistant soybeans, soybean cyst nematode resistant soybeans and methods of breeding and identifying resistant plants. US Patent US6300541 B1

Lorenz A, Smith K, Jannink J (2012) Potential and optimization of genomic selection for Fusarium head blight resistance in six-row barley. Crop Sci 52:1609-1621

Lorenzana RE, Bernardo R (2009) Accuracy of genotypic value predictions for marker-based selection in biparental plant populations. Theor Appl Genet 120:151-161

Luckew AS, Cianzio SR, Leandro LF (2012) Screening method for distinguishing soybean resistance to in resistant $\times$ resistant crosses. Crop Sci 52:2215-2223

Luckew A, Leandro L, Bhattacharyya M, Nordman D, Lightfoot D, Cianzio S (2013) Usefulness of 10 genomic regions in soybean associated with sudden death syndrome resistance. Theor Appl Genet 126(9):2391-2403. doi:10. 1007/s00122-013-2143-4

Malvick D, Bussey K (2008) Comparative analysis and characterization of the soybean sudden death syndrome pathogen Fusarium virguliforme in the northern United States. Can J Plant Pathol 30:467-476

Mamidi S, Chikara S, Goos RJ, Hyten DL, Annam D, Moghaddam SM, Lee RK, Cregan PB, McClean PE (2011) Genome-wide association analysis identifies candidate genes associated with iron deficiency chlorosis in soybean. Plant Genome 4:154-164

Mamidi S, Lee RK, Goos JR, McClean PE (2014) Genome-wide association studies identifies seven major regions responsible for iron deficiency chlorosis in soybean (Glycine max). PLoS One 9(9):e107469. doi:10.1371/journal.pone. 0107469

Meksem K, Doubler T, Chancharoenchai K, Nijti N, Chang S, Arelli AR, Cregan P, Gray L, Gibson P, Lightfoot D (1999) Clustering among loci underlying soybean resistance to Fusarium solani, SDS and SCN in near-isogenic lines. Theor Appl Genet 99:1131-1142

Meuwissen T, Goddard M (2010) Accurate prediction of genetic values for complex traits by whole-genome resequencing. Genetics 185:623-631

Mueller D, Hartman G, Nelson R, Pedersen W (2002) Evaluation of Glycine max germ plasm for resistance to Fusarium solani $\mathrm{f}$. sp. glycines. Plant Dis 86:741-746

Mueller D, Nelson R, Hartman G, Pedersen W (2003) Response of commercially developed soybean cultivars and the ancestral soybean lines to Fusarium solani $\mathrm{f}$. sp. glycines. Plant Dis 87:827-831

Navi SS, Yang X (2008) Foliar symptom expression in association with early infection and xylem colonization by Fusarium virguliforme (formerly $F$. solani $\mathrm{f}$. sp. glycines), the causal agent of soybean sudden death syndrome. Plant Health. doi:10.1094/PHP-2008-0222-01-RS

Njiti V, Doubler T, Suttner RJ, Gray L, Gibson P, Lightfoot D (1998) Resistance to soybean sudden death syndrome and root colonization by Fusarium solani f. sp. glycine in nearisogenic lines. Crop Sci 38:472-477

Njiti V, Johnson J, Torto T, Gray L, Lightfoot D (2001) Inoculum rate influences selection for field resistance to soybean sudden death syndrome in the greenhouse. Crop Sci 41:1726-1731

Njiti V, Meksem K, Iqbal M, Johnson J, Kassem MA, Zobrist K, Kilo V, Lightfoot D (2002) Common loci underlie field resistance to soybean sudden death syndrome in forrest, pyramid, essex, and douglas. Theor Appl Genet 104:294-300

Prabhu R, Njiti V, Bell-Johnson B, Johnson J, Schmidt M, Klein J, Lightfoot D (1999) Selecting soybean cultivars for dual resistance to soybean cyst nematode and sudden death syndrome using two DNA markers. Crop Sci 39:982-987

Pritchard JK, Stephens M, Rosenberg NA, Donnelly P (2000) Association mapping in structured populations. Am J Human Genet 67:170-181

R Development Core Team (2005) R: a language and environment for statistical computing. Vienna, Austria. http:// www.r-project.org. Accessed 25 April 2014

Rafalski A (2002) Applications of single nucleotide polymorphisms in crop genetics. Curr Opin Plant Biol 5:94-100 
Roy K, Hershman D, Rupe J, Abney T (1997) Sudden death syndrome of soybean. Plant Dis 81:1100-1111

Rutkoski J, Benson J, Jia Y, Brown-Guedira G, Jannink J, Sorrells M (2012) Evaluation of genomic prediction methods for fusarium head blight resistance in wheat. Plant Genome 5:51-61

Rutkoski J, Poland J, Singh R, Huerta-Espino J, Bhavani S, Barbier H, Rouse M, Jannink J, Sorrells M (2014) Genomic selection for quantitative adult plant stem rust resistance in wheat. Plant Genome. doi:10.3835/plantgenome2014.02. 0006

Sanogo S, Yang X (2001) Relation of sand content, pH, and potassium and phosphorus nutrition to the development of sudden death syndrome in soybean. Can J Plant Pathol 23:174-180

Schmidt M, Suttner R, Klein J, Gibson P, Lightfoot D, Myers O Jr (1999) Registration of LS-G96 soybean germplasm resistant to soybean sudden death syndrome and soybean cyst nematode race 3. Crop Sci 39(2):598. doi:10.2135/ cropsci1999.0011183X003900020072x

Song Q, Hyten DL, Jia G, Quigley CV, Fickus EW, Nelson RL, Cregan PB (2013) Development and evaluation of SoySNP50K, a high-density genotyping array for soybean. PLoS One 8:e54985

Sukumaran S, Xiang W, Bean SR, Pedersen JF, Kresovich S, Tuinstra MR, Tesso TT, Hamblin MT, Yu J (2012) Association mapping for grain quality in a diverse sorghum collection. Plant Genome 5:126-135

Technow F, Bürger A, Melchinger AE (2013) Genomic prediction of northern corn leaf blight resistance in maize with combined or separated training sets for heterotic groups. G3: Genes|Genomes|Genetics 3(2):197-203

Triwitayakorn K, Njiti V, Iqbal M, Yaegashi S, Town C, Lightfoot D (2005) Genomic analysis of a region encompassing QRfs1 and QRfs2: genes that underlie soybean resistance to sudden death syndrome. Genome 48:125-138

Vick C, Chong S, Bond J, Russin J (2003) Response of soybean sudden death syndrome to subsoil tillage. Plant Dis 87:629-632

Wang S, Dvorkin D, Da Y (2012) SNPEVG: a graphical tool for GWAS graphing with mouse clicks. BMC Bioinformatics 13:319

Wen Z, Tan R, Yuan J, Bales C, Du W, Zhang S, Chilvers M, Schmidt C, Song Q, Cregan P, Wang D (2014) Genomewide association mapping of quantitative resistance to sudden death syndrome in soybean. BMC Genom 15:809

Wrather J, Koenning S (2009) Effects of diseases on soybean yields in the United States 1996 to 2007. Plant Health Progress. doi:10.1094/PHP-2009-0401-01-RS

Zhou H, Steffenson B (2013) Genome-wide association mapping reveals genetic architecture of durable spot blotch resistance in US barley breeding germplasm. Mol Breed 32:139-154

Zhou H, Steffenson B, Muehlbauer G, Wanyera R, Njau P, Ndeda S (2014) Association mapping of stem rust race TTKSK resistance in US barley breeding germplasm. Theor Appl Genet 127(6):1293-1304. doi:10.1007/ s00122-014-2297-8 